23

\title{
Спектральные характеристики наклонного отражательного интерферометра как сенсора показателя преломления
}

\author{
(C) В.С. Терентьев, В.А. Симонов \\ Институт автоматики и электрометрии Сибирского отделения РАН, \\ 630090 Новосибирск, Россия \\ e-mail: terentyev@iae.nsk.su
}

Поступила в редакцию 15.09.2020 г.

В окончательной редакции 12.10.2020 г.

Принята к публикации 14.10.2020 г.

\begin{abstract}
Впервые представлено моделирование сенсора показателя преломления анализируемой жидкости в конфигурации Кречмана на основе наклонного отражательного интерферометра (ОИ) и исследованы его спектральные свойства. Принцип работы данного сенсора основан на эффекте инвертированного поверхностного плазмонного резонанса (ИППР). Чувствительной структурой является металл-диэлектрическое многослойное покрытие на основе никелевой тонкой пленки в сочетании с нечетвертьволновыми диэлектрическими слоями. Описано моделирование процесса изготовления ОИ при наклонном падении света. Приведены формулы для оценки чувствительности, спектральной ширины максимума отражения ИППР, а также параметра качества. Показано, что за счет высокой добротности данный тип сенсора может иметь очень большие параметры качества $\left(>10^{3}\right)$.
\end{abstract}

Ключевые слова: отражательный интерферометр, полное внутреннее отражение, сенсор показателя преломления.

DOI: $10.21883 /$ OS.2021.02.50564.232-20

\section{Введение}

Сенсоры на основе эффекта нарушенного полного внутреннего отражения или, другими словами, на основе возбуждения поверхностного плазмонного резонанса (ППР) широко применяются для детектирования изменения показателя преломления анализируемых жидкостей (аналитов) в биологии [1]. Одна из стандартных и широко распространенных схем для сенсоров ППР основана на конфигурации Кречмана (рис. 1), в которой присутствуют призма и нанесенная на ее грань чувствительная структура (SS). Вариантом SS может быть тонкая металлическая пленка $(\leq 50 \mathrm{~nm})$. В видимом и ближнем ИК спектральных диапазонах наилучшим образом для этого подходят благородные металлы золото и серебро [2]. Также могут использоваться и метаматериалы, состоящие из наноструктурированных объектов [3]. При падении света на такую структуру под углом, большим, чем угол полного внутреннего отражения (ПВО, TIR) системы призма-аналит, в спектре отраженного излучения получаются узкие резонансные минимумы интенсивности, спектральное положение которых соответствует условию возбуждения ППР. При этом ППР будет как в спектральной области, так и в зависимости от угла падения света. При изменении показателя преломления аналита положение ППР будет сдвигаться (по длине волны или углу). Как и для любого сенсора, можно определить три основные характеристики сенсоров показателя преломления - разрешающая способность, чувствительность и параметр качества. Разрешающая способность $\delta n_{a}$ в том числе определяется шумами измерительной схемы: источника и приемника излучения, температурной и вибрационной стабильностью оптической схемы, электроники и т.п., a также шириной ППР на полувысоте измеряемой величины, например длины волны $\delta \lambda$ или угла падения $\delta \alpha$. В данной работе будут исследованы спектральные характеристики сенсора, где величина $\delta \lambda$ имеет размерность нанометров $[\mathrm{nm}]$. Чувствительность спектрального сенсора $S_{\lambda}$, которая определяет изменение (смещение) интенсивности спектральной характеристики по длине волны от изменения показателя преломления (RIU) аналита, имеет размерность [nm/RIU]. Кроме этого, для сравнительного показателя качества (figure of merit, FOM) сенсоров можно использовать величину $\mathrm{FOM}=S_{\lambda} / \delta \lambda\left[\mathrm{RIU}^{-1}\right]$ [4]. Характеристики сенсоров могут быть улучшены за счет использования дополнительных диэлектрических слоев [5]. В работах [6,7] была продемонстрирована металл-диэлектрическая структура из металлической пленки и диэлектрического многослойного покрытия, которая имеет узкий спектральный ППР $(5 \mathrm{~nm})$ для $S$-поляризованного света, причем при использовании пленки из неблагородного металла. Многослойное диэлектрическое покрытие создает в структуре условия для многолучевой интерференции, что существенно повышает разрешающую способность сенсора по сравнению с простой структурой, состоящей только из металлического слоя или из металла и одного диэлектрического слоя.

Кроме сенсоров на основе резонансных потерь в интенсивности при возбуждении ППР существуют сенсоры на основе инвертированного поверхностного плазмон- 


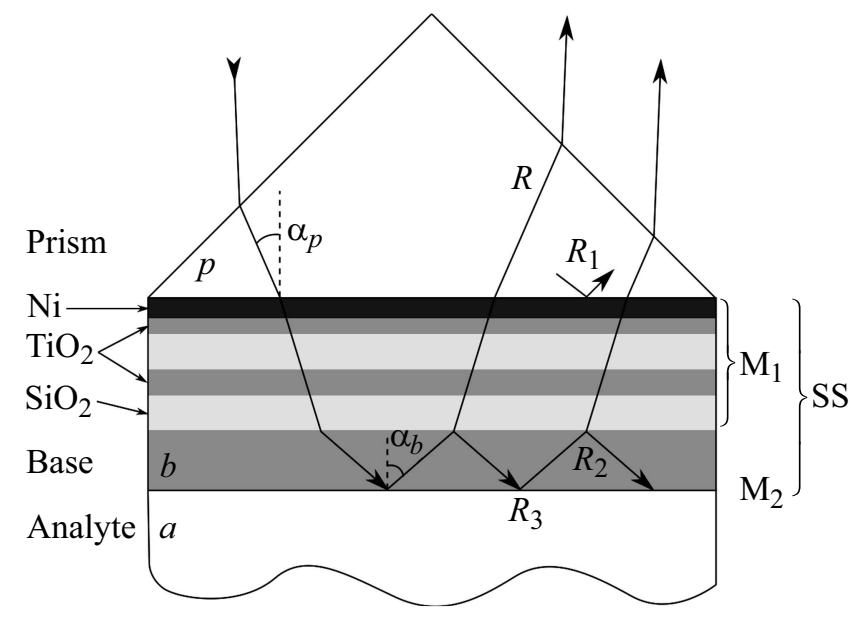

Рис. 1. Оптическая схема сенсора на основе наклонного ОИ: SS - чувствительная структура, $p$, Prism - призма $\left(n_{p}=1.74\right), \mathrm{Ni}-$ никелевая пленка толщиной $6 \mathrm{~nm}$ $\left(n_{\mathrm{Ni}}=3.4378-6.7358 i\right)$, многослойное диэлектрическое покрытие из оксида титана $\mathrm{TiO}_{2}\left(n_{\mathrm{TiO}_{2}}=2.4\right)$, оксида кремния $\mathrm{SiO}_{2} \quad\left(n_{\mathrm{SiO}_{2}}=1.46\right) ; \quad(b)$ Base - базовый слой $\left(n_{b}=2.4\right)$, (a) Analyte - аналит $\left(n_{a}=1.33\right), \alpha_{p}-$ угол падения в призме, $\alpha_{b}$ - угол падения в базовом слое, $R_{1,2}$ - коэффициенты отражения зеркала $\mathrm{M}_{1}, R_{3}$ - зеркала $\mathrm{M}_{2}$.

ного резонанса (ИППР) [8-10]. Данный тип сенсоров имеет узкий резонансный максимум в отраженном свете. Структура ИППР сенсора настроена так, что вне резонанса вся или большая часть падающей световой энергии поглощается или рассеивается, но в области резонанса потери резко уменьшаются и появляется максимум отражения. Такие свойства инвертированы относительно свойств ППР-сенсоров, в которых структура наоборот настроена на отражение вне резонанса, а ППР соответствует увеличению потерь. Сенсоры ИППР имеют те же характеристики, что и ППР-сенсоры, к ним применимы такие же критерии разрешающей способности $\delta n_{a}$, спектральной ширины ИППР $\delta \lambda$, чувствительности $S_{\lambda}$ и показателя качества FOM. При этом ИППР-сенсоры могут иметь существенное преимущество перед традиционными ППР-сенсорами из-за возможности использования их в лазерном резонаторе для создания положительной обратной связи, что может значительно сузить $\delta \lambda$ ИППР и за счет этого повысить FOM. Особенно большой выигрыш получается при одночастотной генерации в волоконном лазере с кольцевым резонатором, но для этого необходимая спектральная ширина ИППР должна быть около 100 pm [11]. Однако в настоящее время рассматриваемые ИППР-сенсоры в схеме Кречмана имеют значительно большие ширины резонансов (более $10 \mathrm{~nm}$ ), так как в них используются либо только один слой металла, либо к нему добавляется один или два диэлектрических слоя $[2,10]$.

В настоящей работе предлагается использовать отражательный интерферометр (ОИ) для создания ИППРсенсора. Отражательный интерферометр - это двухзер- кальный интерферометр с резонатором типа интерферометра Фабри-Перо (ИФП), однако имеющий более сложную структуру зеркал. Переднее зеркало ОИ, на которое падает свет, может иметь асимметрию коэффициентов отражения с разных сторон, обусловленную потерями в структуре, тогда как заднее зеркало обычное, без потерь. Если асимметрия коэффициентов отражения переднего зеркала очень велика, а коэффициент отражения заднего зеркала очень близок единице, то возможно получить в отраженном свете так называемую „необращенную“ аппаратную функцию, подобную аппаратной функции ИФП в пропускании. Такую узкополосную селекцию излучения в отражении выгодно использовать в лазерных резонаторах для селекции излучения [12]. До сего времени ОИ применялись только для нормального падения света и были разработаны как в объемных [13], так и в волоконных вариантах $[14,15]$. Исследование характеристик ОИ с необращенной аппаратной функцией для наклонного падения света, насколько известно авторам, еще не проводилось.

\section{Теория наклонного ОИ}

Для описания параметров сенсора ИППР на основе ОИ целесообразнее всего воспользоваться разработанной для него теорией в приближении плоских волн. Это обусловлено тем, что в данной задаче присутствует значительно более сложная структура покрытия, чем для обычных ИППР- или ППР-сенсоров. В ней, помимо поглощающего металлического слоя, присутствуют нечетвертьволновые многослойные покрытия, и с помощью данной теории можно достаточно простыми выражениями аналитически описать характеристики сенсора.

Известно, что аппаратная функция ОИ, справедливая и для наклонного падения света, в приближении плоских волн описывается формулами

$$
\begin{gathered}
R=R_{1}-\frac{\sqrt{R_{1} R_{2}} 2 R_{3} T_{1} \cos (\vartheta)+2 T_{1} \sqrt{R_{1} R_{3}} \cos (\vartheta+2 \varphi)}{1+R_{3} R_{2}-2 \cos (2 \varphi) \sqrt{R_{3} R_{2}}} \\
+\frac{R_{3} T_{1}^{2}}{1+R_{3} R_{2}-2 \cos (2 \varphi) \sqrt{R_{3} R_{2}}} \\
\vartheta=\Psi_{1}+\Psi_{2}-2 \Phi_{1} \\
\varphi(\alpha, \lambda)=\frac{2 \pi n_{b} L_{b} \cos (\alpha)}{\lambda}-\frac{\Psi_{2}(\alpha, \lambda)+\Psi_{3}(\alpha, \lambda)}{2}
\end{gathered}
$$

где $\lambda$ - длина волны, $\alpha$ - угол падения света на зеркала, $\Psi_{1,2} \Phi_{1}-$ фазы коэффициентов отражения и пропускания (соответственно $R_{1,2}$ и пропускания $T_{1}=T_{2}$ ) зеркала $\mathrm{M}_{1} ; \Psi_{3}$ и $R_{3}$ - фаза и коэффициент отражения заднего зеркала $\mathrm{M}_{2}, L_{b}$ - толщина, $n_{b}$ - показатель преломления базы ОИ (рис. 1). Как видно из (1), коэффициент отражения представляет собой сложную функцию, состоящую из трех членов. Это обусловлено двухлучевой интерференцией первого отраженного луча (с амплитудой $\sqrt{R_{1}}$ ) и суммарного поля, выходящего 
из ОИ. В то же время в пропускании ОИ, как и ИФП, имеется только один член, с точностью до константы пропорциональный третьему члену (1). Первые два члена в (1) стремятся к нулю, если $R_{1} \rightarrow 0$. В этом случае зависимость $R(\varphi)$ с точностью до константы будет подобна пропусканию ИФП (необращенная форма аппаратной функции). Если же $R_{1} \neq 0$ и $\varphi \neq(2 m+1) \pi$ ( $m$ - целое), что характерно для систем с потерями, то форма профиля $R(\varphi)$ будет асимметрична (в пропускании ИФП форма профиля симметрична).

Из (1) можно с достаточно хорошей точностью определить резкость полос $F$ при $R_{1} \approx 0$ :

$$
F \approx \pi \frac{\sqrt[4]{R_{2} R_{3}}}{1-\sqrt{R_{2} R_{3}}} \underset{\left(R_{3}=1\right)}{=} \pi \frac{\sqrt[4]{R_{2}}}{1-\sqrt{R_{2}}}
$$

и максимальный коэффициент отражения $R_{\max }$ :

$$
R_{\max } \approx \frac{\left(1-R_{2}\right)^{2} R_{3}}{4\left(1-\sqrt{R_{2} R_{3}}\right)^{2}} \underset{\left(R_{3}=1\right)}{=} \frac{\left(1+\sqrt{R_{2}}\right)^{2}}{4} .
$$

В настоящей работе проводится моделирование наклонного ОИ, состоящего из переднего зеркала $\mathrm{M}_{1}$, образованного металлической пленкой $\mathrm{Ni}$, согласованным диэлектрическим многослойным покрытием, диэлектрическим слоем базы и задним зеркалом $\mathrm{M}_{2}$, которое представляет собой границу раздела базы с аналитом (Analyte) (рис. 1). ОИ должен иметь высокий, узкий спектральный максимум интенсивности в отражении при падении света под углом $\alpha_{p}$ на грань призмы в условиях ПВО (TIR) системы призма-аналит, т. е. $\alpha_{p}>\alpha_{p}^{\mathrm{TIR}}=\arcsin \left(n_{a} / n_{p}\right)$, где $n_{a}-$ показатель преломления аналита, $n_{p}-$ призмы. Для этого, согласно (1), (3), (4), $R_{1} \rightarrow 0, R_{2,3} \rightarrow 1$, при этом $R_{2}<R_{3}$. $\mathrm{B}$ условиях ПВО и при отсутствии потерь на рассеяние и поглощение коэффициент отражения заднего зеркала $R_{3}=1$, и можно записать более упрощенные формулы (3) и (4) (после знака равенства), при этом у зеркала $\mathrm{M}_{2}$ при изменении $n_{a}$ изменяется только фаза отражения $\Psi_{3}$. Аналитическое выражение этой фазы можно получить из формул Френеля для амплитуды коэффициента отражения от границы раздела базы и аналита. Например, для $S$-поляризованного света ее можно выразить через угол $\alpha_{p}$ падения в призме, показатели преломления $n_{p}, n_{a}$ и $n_{b}$ :

$$
\operatorname{tg}\left(\Psi_{3}\right)=\frac{2 \sqrt{n_{b}^{2}-\left(n_{p} \sin \left(\alpha_{p}\right)\right)^{2}} \sqrt{\left(n_{p} \sin \left(\alpha_{p}\right)\right)^{2}-n_{a}^{2}}}{n_{b}^{2}+n_{a}^{2}-2\left(n_{p} \sin \left(\alpha_{p}\right)\right)^{2}} .
$$

Используя (2) и (5), можно вычислить производную $\varphi$ по $n_{a}$ :

$$
\begin{aligned}
\frac{\partial \varphi}{\partial n_{a}}= & -\frac{1}{2} \frac{\partial \Psi_{3}}{\partial n_{a}}=\frac{n_{a} \sqrt{n_{b}^{2}-\left(n_{p} \sin \left(\alpha_{p}\right)\right)^{2}}}{n_{b}^{2}-n_{a}^{2}} \\
& \times \frac{1}{\sqrt{\left(n_{p} \sin \left(\alpha_{p}\right)\right)^{2}-n_{a}^{2}}} .
\end{aligned}
$$

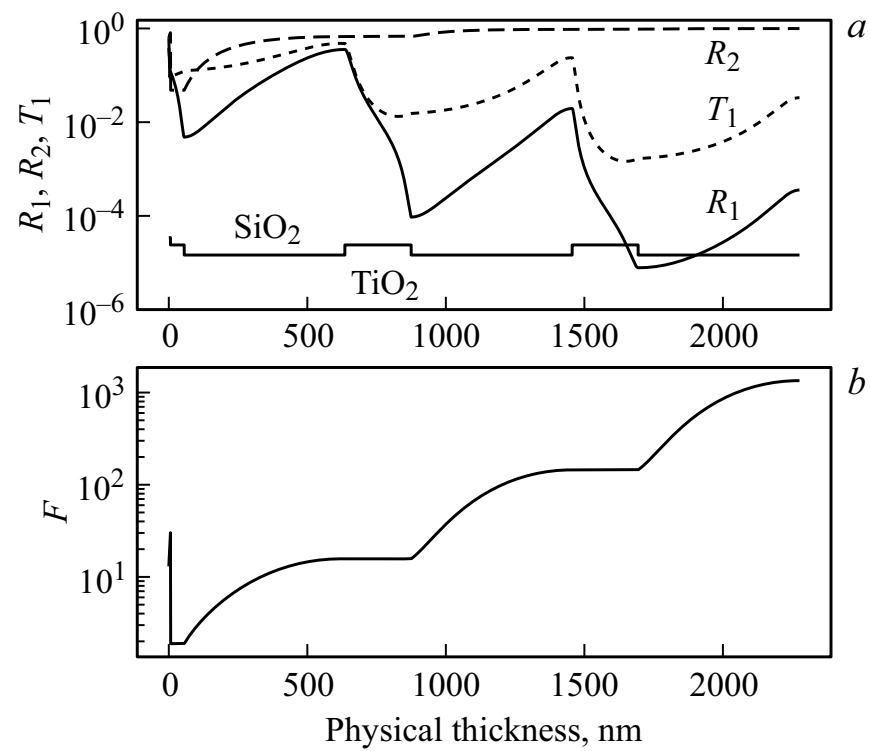

Рис. 2. Моделирование процесса нанесения металлдиэлектрического покрытия на грань призмы в конфигурации Кречмана: (a) $R_{1}$ - коэффициент отражения в призме, $T_{1}-$ пропускание зеркала $\mathrm{M}_{1}$, когда выходной средой является аналит, $R_{2}$ - коэффициент отражения зеркала $\mathrm{M}_{1}$ с обратной стороны, когда аналит заменен на базовый слой, коэффициенты приведены для $S$-поляризованного света, снизу линией показано распределение показателя преломления оксида титана и оксида кремния, угол падения $a_{p}^{\prime}=49.5^{\circ}$. (b) Зависимость резкости полос $F$ от толщины многослойного покрытия.

Формула (6) дает возможность оценить чувствительность сенсора, при этом ее величину можно варьировать на порядки за счет подкоренного выражения в знаменателе путем приближения $\alpha_{p}$ к углу $\alpha_{p}^{\mathrm{TIR}}$.

\section{Метод получения необращенной картины при наклонном падении}

Метод получения необращенной интерференционной картины (аппаратной функции) в отражении от ОИ при нормальном падении света на основе тонкой металлической пленки описан в [14]. Он заключается в контроле процесса формирования (напыления) зеркала $\mathrm{M}_{1}$ по коэффициенту отражения $R_{1}$ со стороны подложки от структуры, состоящей из подложки, металлической пленки, последовательно наносимых диэлектрических покрытий и смене диэлектриков в экстремальных точках. При этом происходит постепенное подавление отражения в подложку $\left(R_{1}\right)$ и рост отражения с обратной стороны $\left(R_{2}\right)$. Затем, если добавить к данному зеркалу базу определенной длины и заднее высокоотражающее зеркало, то в отражении получается необращенная аппаратная функция. Однако данный метод был разработан только для нормального падения света.

Для наклонного падения результат расчета представлен на рис. 2, при этом возникают несколько нюансов, 
и один из них состоит в выборе поляризации света. Как показало моделирование параметров зеркала $\mathrm{M}_{1}$, подавление $R_{1}$ и рост $R_{2}$ были обнаружены только для $S$-поляризации, тогда как сенсоры ППР обычно работают при $P$-поляризованном свете. Другой нюанс состоит в выборе угла падения и связанными с этим спектральными свойствами $R_{2}$. Известно, что максимум спектрального коэффициента отражения многослойного диэлектрического зеркала смещается по спектру в более коротковолновую область при увеличении угла падения. Чтобы получить максимум отражения на целевой длине волны и под углом, необходимо увеличить толщину слоев многослойного покрытия по сравнению со случаем нормального падения. Тогда при $R_{3}=1$ (в условиях ПВО), согласно формулам (3), (4), $R_{2}$ будет максимальным, и мы получаем высокую резкость полос $F$.

В методе для нормального падения выходной средой служит воздух, однако при наклонном падении для углов, превышающих $\alpha_{\mathrm{Air}}^{\mathrm{TIR}}=\arcsin \left(1 / n_{p}\right)$, возникнет ПВО, и для всех больших углов метод контроля по отражению становится неприменим. То же самое будет, если выходная среда - аналит с $n_{a}>1$ и $\alpha_{p}>\alpha_{p}^{\mathrm{TIR}}$. Учитывая данные нюансы, в моделировании контролировался процесс нанесения диэлектрических слоев под углом $\alpha_{p}^{\prime}$, меньшим и очень близким к углу ПВО: $0<\alpha_{p}^{\mathrm{TIR}}-\alpha_{p}^{\prime}<10^{-2} \mathrm{rad}$. При таком угле падения становится возможным контролировать процесс синтеза покрытия по отражению $R_{1}$, и будет формироваться структура слоев с максимально высоким $R_{2}$ для этого угла падения и целевой (контрольной) длины волны, и, что самое важное, это будет справедливо и для некоторой небольшой окрестности углов падения относительно данного угла. Угол $\alpha_{p}^{\prime}$ нужен только для расчета структуры зеркала $\mathrm{M}_{1}$, но для работы сенсора необходимо сместиться за угол ПВО, т.е. необходимо, чтобы угол падения $\alpha_{p}>\alpha_{p}^{\mathrm{TIR}}$. Для небольших углов смещения от $\alpha_{p}^{\prime}$ максимум спектрального распределения отражения $R_{2}$ также будет мало смещаться по спектру и мало меняться для целевой длины волны, особенно если использовать чередующиеся слои с большой разницей показателей преломления $(\approx 1)$, которые создают большой спектральный диапазон высокого отражения.

Основываясь на этих предположениях, в моделировании было принято, что выходной средой является аналит. Практически такой вариант изготовления реализовать достаточно сложно, по крайней мере заведомо невозможно в рамках вакуумного напыления. Однако с помощью моделирования можно определить толщины слоев, которые можно экспериментально реализовать, например, путем контроля толщины слоев по времени при магнетронном вакуумном напылении. Для демонстрации метода и без потери общности были выбраны показатели преломления призмы $n_{p}=1.74$ и аналита $n_{a}=1.33$. Угол падения в призме $\alpha_{p}^{\prime}=49.5^{\circ}$ был выбран меньше и очень близко к углу $\alpha_{p}^{\mathrm{TIR}}=49.85^{\circ}$ ПВО системы призма-аналит. Выбор такого угла падения обусловлен еще и тем, что выгоднее работать вблизи угла ПВО, так как повышается чувствительность сенсора к изменению фазы отражения для обеих поляризаций. Если по каким-то причинам необходимо моделировать структуры для других углов падения, превышающих и далеко отстоящих от угла ПВО, то, по-видимому, необходимо менять коэффициент преломления выходной среды на больший, чем $n_{a}$, но меньший, чем $n_{p}$.

Если более подробно описывать моделирование процесса изготовления зеркала $\mathrm{M}_{1}$, то оно включает следующую структуру многослойного покрытия. В качестве основы служит конфигурация Кречмана с призмой (рис. 1). На грань призмы наносится тонкий слой металла с толщиной, много меньше длины волны. В данном моделировании был выбран $\mathrm{Ni}$ как наиболее стойкий и имеющий однородную структуру пленки, слабо подверженной влиянию условий изготовления. Выбор неблагородного металла выгодно отличает данный тип сенсора перед сенсорами ППР на основе благородных металлов, так как последние дороже и могут требовать строгого учета. Кроме никеля могут использоваться и другие металлы. Оптимальная толщина пленки металла определяется по критерию согласования [16]:

$$
\xi^{\prime}=n_{p} \cos \left(\alpha_{p}\right)
$$

где $\xi=\xi^{\prime}+i \xi^{\prime \prime}-$ комплексная поверхностная проводимость пленки. В терминах комплексной диэлектрической проницаемости $\varepsilon$ поверхностная проводимость $\xi=i \frac{2 \pi L_{\mathrm{Me}}}{\lambda} \varepsilon\left(\varepsilon=n_{\mathrm{Me}}^{2}\right)$, где $L_{\mathrm{Me}}<\lambda / 20-$ толщина, а $n_{\mathrm{Me}}-$ комплексный показатель преломления металла, $\lambda$ - длина волны света. Толщина металлической пленки никеля с показателем преломления $n_{\mathrm{Ni}}=3.4378-6.7358 i$ на $\lambda=1550 \mathrm{~nm}$ [17], удовлетворяющая (7), составила $L_{\mathrm{Ni}}=6 \mathrm{~nm}$.

Далее на металлическую пленку последовательно наносятся диэлектрические слои, начиная с большего показателя преломления $\mathrm{TiO}_{2}\left(n_{\mathrm{TiO}_{2}}=2.4\right)$. При этом $R_{1}$ достигает минимума при нанесении $\mathrm{TiO}_{2}$ и максимума при нанесении $\mathrm{SiO}_{2}\left(n_{\mathrm{SiO}_{2}}=1.46\right)$, а $R_{2}$ стремится к единице (рис. 2,a). Коэффициент $R_{1}$ рассчитывается для угла падения в призме $\alpha_{p}^{\prime}$, а $R_{2}$ - для угла падения в среду $\alpha_{b}^{\prime}$ с показателем преломления $n_{b}=2.4$ базы, $\sin \left(\alpha_{b}^{\prime}\right)=n_{p} \sin \left(\alpha_{p}^{\prime}\right) / n_{b}=\sin \left(33.46^{\circ}\right)$, а не для аналита, чтобы правильно рассчитывать $F$ в дальнейшем, когда к зеркалу $\mathrm{M}_{1}$ будет добавлена база с $n_{b}=2.4$. Стоит заметить, что по сравнению со случаем нормального падения для получения убывающего $R_{1}$ необходимо увеличить физическую толщину контроля слоев на коэффициент $1 / \cos \left(a_{p}^{\prime}\right)$, при этом максимум спектральной зависимости $R_{2}$ находится на целевой (или вблизи) длине волны $\lambda_{0}=1550 \mathrm{~nm}$.

После расчета структуры зеркала $\mathrm{M}_{1}$ к ней добавляется слой базы (с показателем преломления $n_{b}=2.4$ ) некоторой толщины (большей $\left.\lambda /\left(2 n_{b}\right)\right)$, а выходной средой по- прежнему остается аналит. Теперь при $\alpha_{p}>\alpha_{p}^{\mathrm{TIR}}$ вся структура начинает работать как высокодобротный 
двухзеркальный отражательный интерферометр, так как по сути на границе база-аналит образуется еще одно зеркало - заднее высокоотражающее зеркало $\mathrm{M}_{2}$ (рис. 1). В принципе показатель преломления базы может быть любым. Желательно, чтобы он был как можно больше, чтобы уменьшить угол падения внутри базы и снос пучка при многократных отражениях. Для примера приведем полученные в расчете физические толщины последовательно расположенных слоев, nm: [(Призма), 6, 50.02, 580.49, 239.65, 581.71, 238.53, 582.94, 387.50 , (Аналит)], с соответствующими показателями преломления, RIU: [(1.74), 3.4378-6.7358i, 2.4, 1.46, 2.4, $1.46,2.4,1.46,2.4,(1.33)]$.

Как видно из рис. $2, b$, в данной конфигурации резкость полос $F$, напрямую влияющая на $\delta \lambda$ ИППР, растет, причем только при нанесении слоев $\mathrm{SiO}_{2}$, а слои $\mathrm{TiO}_{2}$ оказывают только фазовые сдвиги для $R_{2}$ (так как выходной средой в данном случае является $\mathrm{TiO}_{2}$ ). Таким образом, даже для 6-слойного покрытия $F$ достигает величины более $10^{3}$ (1347.2). При дальнейшем нанесении слоев резкость $F$ продолжает расти и, повидимому, ограничивается только потерями в слоях покрытия и на границах раздела с аналитом, сносом пучка и нелинейными эффектами в базовом слое из-за мощностных ограничений.

Результирующая спектральная ширина ИППР для последней точки зависимости $F$ показана на рис. $3, a, b$. Толщина базы $L_{b}=0.6 \lambda_{0} / n_{b}$ выбрана минимально возможной, чтобы для угла падения $\alpha_{p}=49.9^{\circ}$ максимум находился около $1530 \mathrm{~nm}$ (подходит для усиления эрбиевым активным волокном). Для данной длины базы (при фиксированном угле падения и длине волны) сенсор обладает чувствительностью, близкой к максимальной. Спектральная ширина на полувысоте ИППР $\delta \lambda=0.8 \mathrm{~nm}$ сравнима с характерной шириной обычной волоконной брэгговской решетки. Значение угла $\alpha_{p}$ было выбрано на основании результатов работы с ППР-сенсором в видимой спектральной области [18], где аналитической средой был газ. Угол $\alpha_{p}$ настолько же близок к углу ПВО, как и в указанной работе. В результате получаются высокие чувствительности и, учитывая конечную угловую расходимость излучения белого света, выходящего из волокна типа SMF-28е и сколлимированного микролинзой, практически реализуемые. Расходимость ограничивает разрешающую способность метода из-за превышения угла ПВО и дополнительного уширения ППР. Данный ППР-сенсор имел чувствительность $(4669 \mathrm{~nm} / \mathrm{RIU})$ и спектральную ширину ППР $\approx 50 \mathrm{~nm}, \mathrm{FOM}=93$.

\section{Характеристики сенсора}

Чтобы получить аналитические формулы для оценки чувствительности $S_{\lambda}$ и разрешающей способности, зависящей от $\delta \lambda$, а также качество FOM сенсора, необходимо оценить область свободной дисперсии $\Delta \lambda$
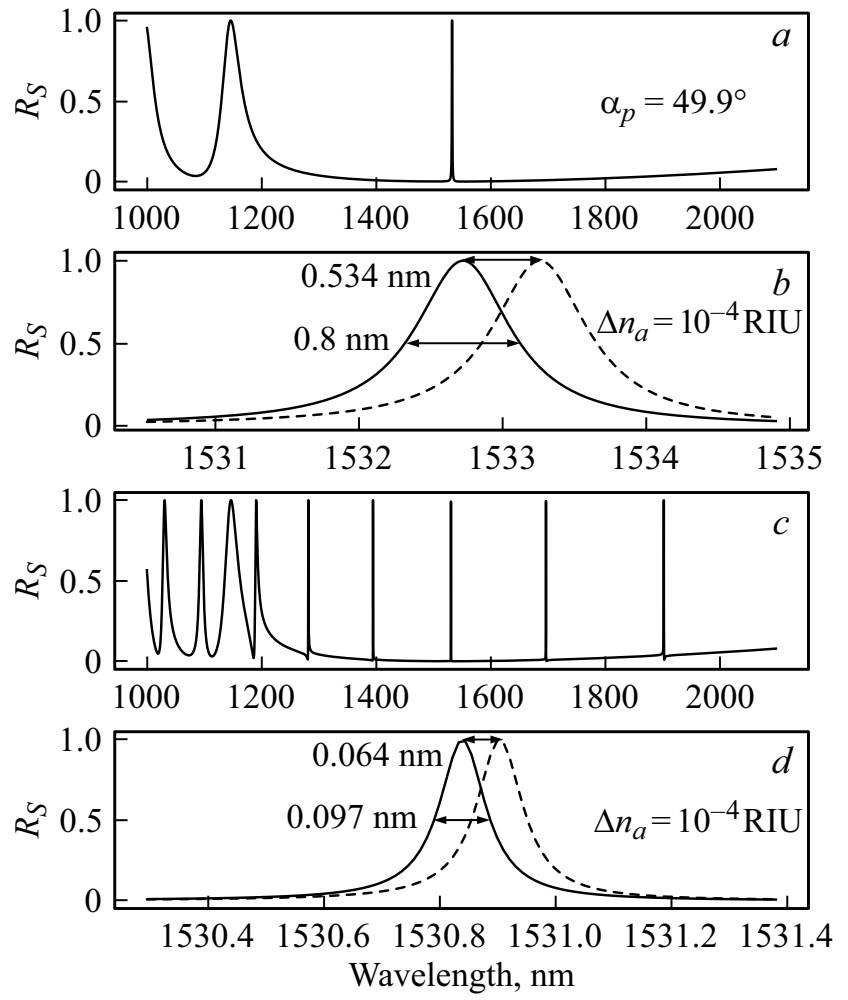

Рис. 3. Спектральные зависимости коэффициента отражения ОИ для $S$-поляризации и угла падения $\alpha_{p}=49.9^{\circ}$ : (a) в широком спектральном диапазоне, (b) в малом спектральном диапазоне около $1530 \mathrm{~nm}$ вблизи максимума отражения при длине базы $L_{b}=0.6 \lambda_{0} / n_{b},(c)$ то же, что и для $(a),(d)$ то же, что и для $(b)$ при длине базы $L_{b}=9.9 \times 0.6 \lambda_{0} / n_{b}$. Штрихами показано спектральное смещение при изменении показателя преломления аналита на $10^{-4} \mathrm{RIU}$.

интерферометра:

$$
\Delta \lambda \approx \frac{\lambda_{0}^{2}}{2\left(n_{b} L_{b} \cos \left(\alpha_{b}\right)+n_{\mathrm{eff}} L_{\mathrm{eff}} \cos \left(\alpha_{\mathrm{eff}}\right)\right)+\lambda_{0}}[\mathrm{~nm}]
$$

где $L_{\text {eff }}-$ эффективная толщина, а $n_{\text {eff }}-$ коэффициент преломления, $\alpha_{\mathrm{eff}}-$ угол падения в зеркале $\mathrm{M}_{1}$. Даже при максимально короткой базе $L_{b}$, при большой разнице в величинах показателей преломления слоев зеркала $\mathrm{M}_{1}$ можно положить $L_{\mathrm{eff}} \approx 0$. Оценочная формула (8) при малых базах может давать ошибку примерно до 1.5 раз, ошибка уменьшается с ростом толщины базы. При изменении фазы отражения $\varphi$ на $\pi$ спектральный максимум отражения ОИ сдвигается на всю область свободной дисперсии в положение соседнего максимума. Отсюда следует формула для спектральной чувствительности:

$$
S_{\lambda}=\frac{\Delta \lambda}{\pi} \frac{\partial \varphi}{\partial n_{a}}=-\frac{\Delta \lambda}{2 \pi} \frac{\partial \Psi_{3}}{\partial n_{a}}\left[\frac{\mathrm{nm}}{\mathrm{RIU}}\right] .
$$

На рис. 4 показана зависимость абсолютного значения относительной чувствительности $\left|S_{\lambda} / \Delta \lambda\right|$ от относительного угла падения в призме $\Delta \alpha_{p}=\alpha_{p}-\alpha_{p}^{\mathrm{TIR}}$ для $n_{p}=1.74, n_{a}=1.33, n_{b}=2.4$. При приближении 


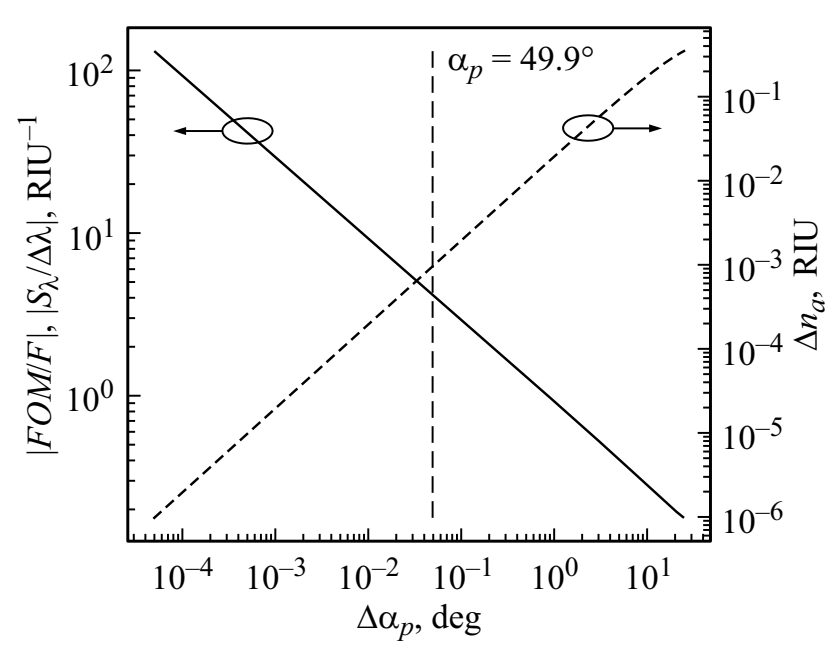

Рис. 4. Зависимости абсолютных значений относительных чувствительности $\left|S_{\lambda} / \Delta \lambda\right|$ и качества $\mathrm{FOM} / F$ сенсора (левая шкала) и динамического диапазона измерения $\Delta n_{a}$ (правая шкала) от относительного угла падения света в призме $\Delta \alpha_{p}=\alpha_{p}-\alpha_{p}^{\mathrm{TIR}}$. Вертикальная штриховая линия показывает характеристики сенсора, показанного на рис. 2.

к углу ПВО чувствительность растет. Вместе с этим снижается динамический диапазон измерения показателя преломления $\Delta n_{a}$ (в большую сторону от показателя преломления аналита):

$$
\Delta n_{a}=n_{p} \sin \left(\alpha_{p}^{\mathrm{TIR}}+\Delta \alpha_{p}\right)-n_{a} .
$$

Разрешающая способность $\delta n_{a}$ сенсора (с учетом всех шумов конкретной измерительной системы) будет зависеть и определяться через спектральную ширину ИППР на полувысоте $\delta \lambda$, которую можно найти по формуле для интерферометра:

$$
\delta \lambda=\frac{\Delta \lambda}{F}[\mathrm{~nm}] .
$$

Качество сенсора с учетом (8) и (9)

$$
\mathrm{FOM}=\left|\frac{S_{\lambda}}{\delta \lambda}\right|=\left|\frac{F}{2 \pi} \frac{\partial \Psi_{3}}{\partial n_{a}}\right|\left[\mathrm{RIU}^{-1}\right] .
$$

График на рис. 4 также можно рассматривать и как значение относительного качества FОМ/F.

Примечательно, что для данного сенсора существует возможность изменять чувствительность $S_{\lambda}$ и $\delta \lambda$ путем кратного изменения длины базы. Это может быть полезно для получения одночастотной генерации в лазерной схеме, если необходимо пожертвовать чувствительностью ради повышения степени селекции. Для примера можно увидеть, как меняются параметры сенсора при увеличении длины базы $L_{b}$ в 9.9 раз (рис. 3, $c, d$ ).

Из формул (8)-(10) для сенсора (рис. 3, $a, b$ ) можно получить оценочные значения характеристик для $\lambda_{0}=1550 \mathrm{~nm}$ : область свободной дисперсии $\Delta \lambda \approx 767 \mathrm{~nm}$, чувствительность $S_{\lambda}=3214 \mathrm{~nm} / \mathrm{RIU}$, $\delta \lambda=0.57 \mathrm{~nm}$ и качество $\mathrm{FOM}=5638 \mathrm{RIU}^{-1}$, что по порядку величины близко к полученным из численного моделирования, т.е. по рис. $3, a, b S_{\lambda}=5340 \mathrm{~nm} / \mathrm{RIU}$, $\delta \lambda=0.8 \mathrm{~nm}, \mathrm{FOM}=6725 \mathrm{RIU}^{-1}$. Для сравнения в [4] приводятся сенсоры с максимальным FОМ около 200, причем обусловлено это ростом чувствительности, а значит, и ростом ограничения на динамический диапазон измерения $n_{a}$. В случае ОИ большие величины FOM достигаются за счет сужения ИППР благодаря многолучевой интерференции света в высокодобротной системе. Это значит, что при одинаковых FOM сенсор на основе данного ОИ может иметь больший динамический диапазон определения $n_{a}$.

\section{Заключение}

В работе представлено моделирование спектрального сенсора показателя преломления анализируемой жидкости в конфигурации Кречмана на основе наклонного ОИ. Приводятся формулы для оценки чувствительности, разрешающей способности, а также параметра качества. Описано моделирование процесса изготовления асимметричного зеркала и наклонного ОИ. Показано, что за счет высокой добротности данный тип сенсора может иметь очень высокие параметры качества FOM.

К недостаткам метода наклонного ОИ можно отнести возможные затруднения при создании условий для высокодобротной многолучевой интерференции изза паразитного рассеяния или поглощения в слоях, особенно в случаях пористых материалов. При очень высокой добротности и/или мощности падающего света могут проявляться эффекты, связанные с нелинейным изменением показателя преломления базы и тепловыми эффектами разогрева окружающей среды. Кроме того, при наклонном падении происходит снос пучка в слое базы, и при высоком коэффициенте отражения $R_{2}$ может проявиться ограничение на количество интерферирующих лучей, т.е. ограничение на максимальную добротность. Для уменьшения влияния данного эффекта необходимо увеличивать ширину пучка, показатель преломления призмы и слоя базы и уменьшать толщину слоя базы.

Преимущества метода заключаются в возможности создания структуры для любого спектрального диапазона, использовании неблагородных металлов, использовании в составе базы чувствительной структуры материалов без значимого поглощения и рассеяния, например субволновых нанофотонных кристаллов [19] для увеличения фазовой чувствительности к параметрам внешней среды, в возможности регулировки спектральной ширины ИППР, возможности использования структуры в схеме с лазерной генерацией, с помощью чего можно на порядки увеличить FOM за счет сужения спектральной ширины ИППР, особенно при получении одночастотной генерации. 
Представляет интерес исследование угловых зависимостей данного сенсора. Они должны иметь не меньший показатель качества, что и для рассмотренных в данной работе спектральных характеристик. Дальнейшие исследования могут быть связаны с альтернативными вариантами структур переднего зеркала ОИ, например с дифракционными металл-диэлектрическими или полностью диэлектрическими структурами вместо тонкой металлической пленки в переднем зеркале, что повысит лучевую стойкость. По возможности целесообразно разработать оптимизированную технологию изготовления с прямым оптическим контролем за параметрами слоев в процессе их нанесения, как для случая нормального падения.

\section{Благодарности}

Выражаем благодарность д.ф.-м.н. Н.Д. Голдиной за подробное и плодотворное обсуждение результатов работы.

\section{Финансирование работы}

Работа выполнена в рамках темы госзадания Института автоматики и электрометрии СО РАН (№ гос. рег. AAAA-A17-117062110026-3).

\section{Конфликт интересов}

Авторы заявляют, что у них нет конфликта интересов.

\section{Список литературы}

[1] Prabowo B.A., Purwidyantri A., Liu K.-C. // Biosensors. 2018. V. 8. N 3. P. 80 . doi $10.3390 /$ bios 8030080

[2] Choi J.-H., Lee J.-H., Son J., Choi J.-W. // Sensors. 2020. V. 20. P. 1003. doi $10.3390 / \mathrm{s} 20041003$

[3] Roh S., Chung T., Lee B. // Sensors. 2011. V. 11. P. 1565. doi 10.3390/s110201565

[4] Shalabney A., Abdulhalim I. // Opt. Lett. 2012. V. 37. N 7. P. 1175. doi 10.1364/OL.37.001175

[5] Zhou Y., Zhang P., He Y., Xu Z., Liu L., Ji Y., Ma H. // Appl. Opt. 2014. V. 53. N 28. P. 6344. doi 10.1364/AO.53.006344

[6] Голдина Н.Д. // Автометрия. 2009. Т. 45. № 6. С. 99; Goldina N.D. // Optoelectron. Instrument. Proc. 2009. V. 45. P. 571. doi 10.3103/S8756699009060120

[7] Голдина Н.Д. Тонкослойные покрытия для лазерной оптики. Новосибирск: Академиздат, 2018. 132 с.

[8] Printz M., Sambles J.R. // J. Mod. Opt. 1993. V. 40. N 11. P. 2095. doi 10.1080/09500349314552131

[9] Shalabney A., Lakhtakia A., Abdulhalim I., Lahav A., Patzig C., Hazek I., Karabchevsky A., Rauschenbach B., Zhang F., $X u$ J. // Photonics and Nanostructures Fundamentals and Appl. 2009. V. 7. P. 176. doi 10.1016/j.photonics.2009.03.003

[10] Boruah R., Mohanta D., Choudhury A., Ahmed G.A. // Opt. Mater. 2015. V. 39. P. 273. doi 10.1016/j.optmat.2014.11.014

[11] Ou P., Jia Y., Cao B., Zhang C., Hu S., Feng D. // Chin. Opt. Lett. 2008. V. 6. P. 845. doi 10.3788/COL20080611.0845
[12] Terentyev V.S., Simonov V.A., Babin S.A. // Laser Phys. Lett. 2017. V. 14. N 2. P. 25103. doi 10.1088/1612-202X/aa548e

[13] Терентьев В.С. // Автометрия. 2009. V. 45. № 6. Р. 89; Terentiev V.S. // Optoelectron. Instrument. Proc. 2009. V. 45. N 6. P. 563. doi 10.3103/S8756699009060119

[14] Terentyev V.S., Simonov V.A., Babin S.A. // Optic Express. 2016. V. 24. N 5. P. 4512. doi 10.1364/OE. 24.004512

[15] Терентьев В.С., Симонов В.А., Лобач И.А., Бабин С.А. // Квант. электрон. 2019. Т. 49. № 4. P. 399; Terentyev V.S., Simonov V.A., Lobach I.A., Babin S.A. // Qvant. Electron. 2019. V. 49. N 4. P. 399. doi 10.1070/QEL16922

[16] Троицкий Ю.В. Одночастотная генерация в газовых лазеpax. Новосибирск: Наука, 1985. 208 с.

[17] Rakić A.D., Djurišic A.B., Elazar J.M., Majewski M.L. // Appl. Opt. 1998. V. 37. P. 5271.

[18] Голдина Н.Д., Терентьев В.С., Симонов В.А. // Опт. и спектр. 2016. Т. 120. № 5. Р. 847. doi 10.7868/S0030403416050111; Goldina N.D., Terent'ev V.S., Simonov V.A. // Opt. Spectrosc. 2016. V. 120. N 5. P. 796. doi 10.1134/S0030400X16050118

[19] Плеханов А.И., Чубаков В.П., Чубаков П.А. // ФТТ. 2011. T. 53. № 6. C. 1081; Plekhanov A.I., Chubakov V.P., Chubakov P.A. // Physics of the Solid State. 2011. V. 53. N 6. P. 1145. doi 10.1134/S1063783411060254 